\title{
Fatal airway obstruction due to a ball-valve clot with identical signs of tension pneumothorax
}

\author{
Hisaaki Munakata', Michiko Higashi' ${ }^{2}$ Takahiro Tamura', Yushi Ueda Adachi' \\ Departments of ${ }^{1}$ Surgical Intensive Care Medicine and ${ }^{2}$ Emergency and Medical Intensive Care Unit, Nagoya University Hospital, Nagoya, Japan
}

Endo-tracheal tube obstruction due to an extensive blood clot is a recognized but very rare complication. A ball-valve obstruction in the airway could function as a check valve for the lung and thorax, resulting in tension pneumothorax-like abnormalities. A 47-year-old female patient had undergone implantation of a left ventricular assist device 3 weeks prior. On postoperative day 17, planned thoracentesis was performed for drainage of a pleural effusion. Despite the drainage, the patient's oxygenation did not improve, and emergency tracheal intubation was conducted. Subsequent computed tomography revealed bilateral pneumothorax. Two days later, the patient's trachea was extubated without complication, and a minitracheostomy tube was placed. Three hours later, reintubation was conducted due to progressive tachypnea. Although successful intubation was confirmed, ventilation became increasingly difficult and finally impossible. Marked increase in pulmonary artery and central venous pressures suggested progression of the previous tension pneumothorax. After emergency extracorporeal membrane oxygenation was initiated, fiberoptic bronchoscopy revealed the presence of a massive clot and ball-valve obstruction of the endotracheal tube. Two weeks later, the patient died due to severe hypoxic brain damage. Diagnosis of ball valve clot is not simple, but intensivists should consider this rare complication.

Key Words: ball valve obstruction; mini-tracheostomy; tension pneumothorax

In clinical settings, tracheal intubation is considered the most reliable airway securing technique for ventilation [1]. Obstruction of the endotracheal tube due to a ball-valve clot is a recognized but very rare complication [2-8]. A ball-valve obstruction in the airway could function as a check valve for the lung and thorax, resulting in tension pneumothorax-like respiratory and circulatory abnormalities. This case report illustrates the difficulty in differentiating tube obstruction and tension pneumothorax.

\section{CASE REPORT}

A 47-year-old female patient who had undergone implantation of a left ventricular-assist device (LVAD; HeartMate II, NIPRO, Osaka, Japan) 3 weeks previously had received noninvasive positive pressure ventilation (NIPPV) for oxygenation. On postoperative day 17 , a planned thoracentesis was performed for drainage of a pleural effusion of the right thorax. Despite removal of the effluent, the patient's oxygenation worsened, and emergency tracheal intubation was conducted. A subsequent emergency computed tomography (CT) revealed right

\section{Case Report}

Received: May 25, 2019

Revised: September 23, 2019

Accepted: November 1, 2019

Corresponding author Yushi Ueda Adachi Department of Surgical Intensive Care Medicine, Nagoya University Hospital, 65 Tsurami-cho, Showa-ku, Nagoya 466-8550, Japan

Tel: +81-527442340

Fax: +81-527442342

E-mail:yuadachi@med.nagoya-u.ac.jp

Copyright (๑) 2020 The Korean Society of Critical Care Medicine

This is an Open Access article distributed under the terms of Creative Attributions Non-Commercial License (http:// creativecommons.org/li-censes/by-nc/4.0/) which permits unrestricted noncommercial use, distribution, and reproduction in any medium, provided the original work is properly cited. 
pneumothorax and left hemothorax (Figure 1). The surgeons speculated that both lower lung lobes may have been injured during implantation of the LVAD, and the chest tubes were replaced.

However, the subsequent mechanical ventilation was unstable because of increasing airway pressure. Fiberoptic bronchoscopy revealed an obstructing clot in the tracheal tube. The clot was removed by a large-bore suctioning catheter (Figure 2), and mechanical ventilation was safely continued.

Two days later, anticoagulation therapy continued with oral administration of $1.5 \mathrm{mg}$ warfarin, and the value of prothrombin international normalized ratio was 1.71 . The activated partial thrombin time was 39.1 seconds. The patient's trachea was extubated without complication. Immediately after extubation, NIPPV was applied again. After the indicated level of coagulation was confirmed, a percutaneous drainage system, Mini-Trach 2 (Smith Medical International, Ashford, UK), was placed in the neck for suctioning of the discharge in the trachea. Slight bloody sputum was suctioned every 15 minutes. However, the patient developed tachypnea (more than 20 breaths/min), and mild desaturation events followed. Progressive dyspnea and tachycardia suggested respiratory failure. Three hours after extubation, the intensive care team elected to reintubate the patient.

Successful intubation was confirmed by chest wall movement, auscultation, and detection of expired carbon dioxide. However, ventilation using Jackson-Rees circuit became progressively difficult and finally impossible. The mean pulmonary arterial and central venous pressures markedly increased to $39 \mathrm{~mm} \mathrm{Hg}$ and $24 \mathrm{~mm} \mathrm{Hg}$, respectively, within 3 minutes. The mean arterial blood pressure and pulse oximetry decreased to $26 \mathrm{~mm} \mathrm{Hg}$ and less than $80 \%$, respectively. Tension pneumothorax was suspected, and emergency bilateral thoracen-

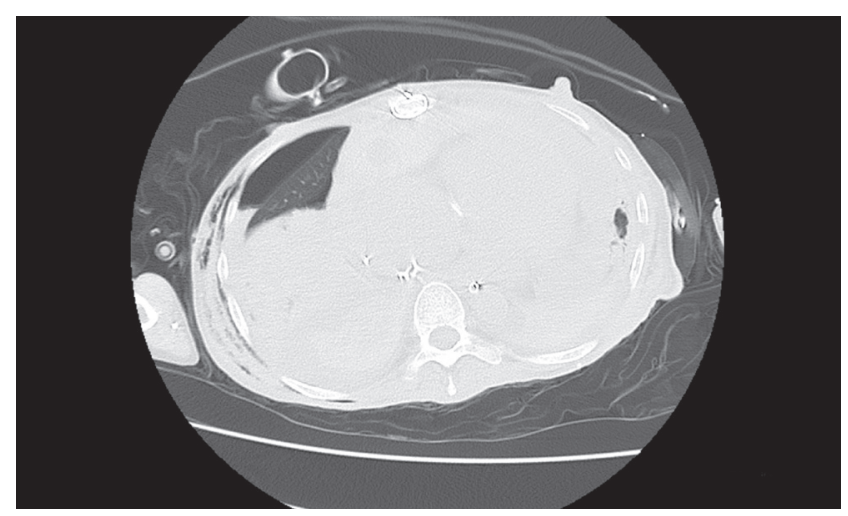

Figure 1. Computed tomogram immediately after the first intubation revealed right pneumothorax and left hemothorax. teses were performed; bloody and foamy air spouted from the needles.

Although the arterial blood pressure immediately increased after the thoracenteses and administration of epinephrine, ventilation was still impossible. HeartMate II, the LVAD, continued pumping blood without failure. The cardiovascular physicians and the engineering team established venous-arterial extracorporeal membrane oxygenation (VA-ECMO) within 15 minutes from the emergency call for the ventilatory failure. The cardiovascular status promptly stabilized, and oxygenation was fully preserved. Successive fiberoptic bronchoscopy revealed obstruction of the trachea by a large blood clot, and suctioning with a large-bore catheter failed to aspirate the clot. Finally, the clot was removed with the endotracheal tube as extubation with suctioning caused the clot to be bonded with the tube. The clot was approximately $1 \mathrm{~cm}$ in diameter and $4 \mathrm{~cm}$ in length (Figure 3). An emergency CT revealed slight pneumothorax and subcutaneous emphysema (Figure 4). Collapse of ventilation was a result of endotracheal

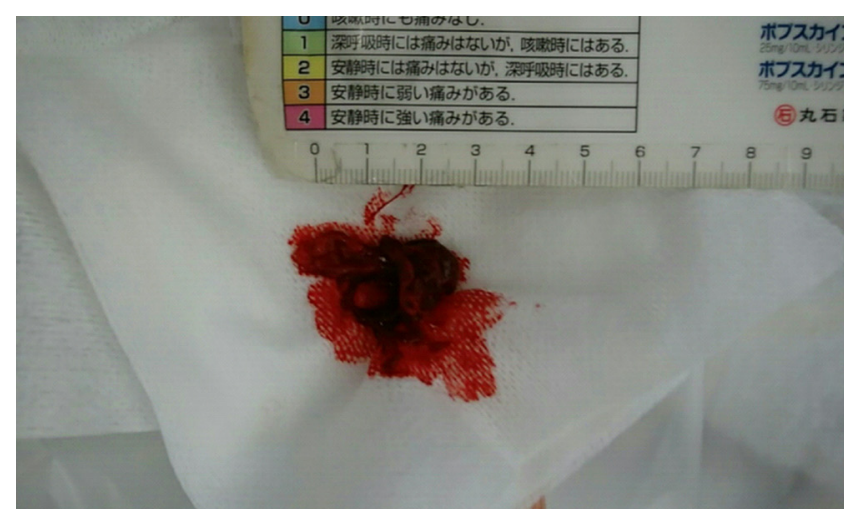

Figure 2. The clot removed after the first ventilation failure. The clot was aspired by a large-bore suction catheter.

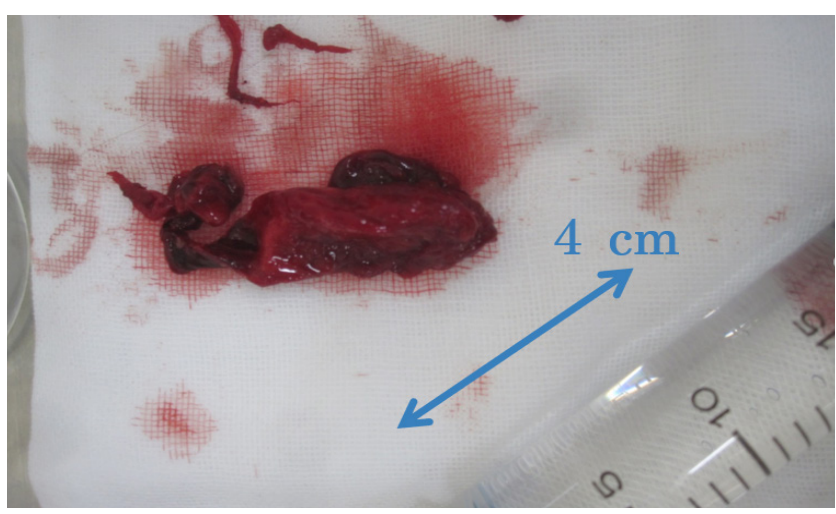

Figure 3. The fatal, obstructing, ball-valve clot that was removed after the second failed ventilation. The clot was approximately 1 $\mathrm{cm}$ in diameter and $4 \mathrm{~cm}$ in length. 


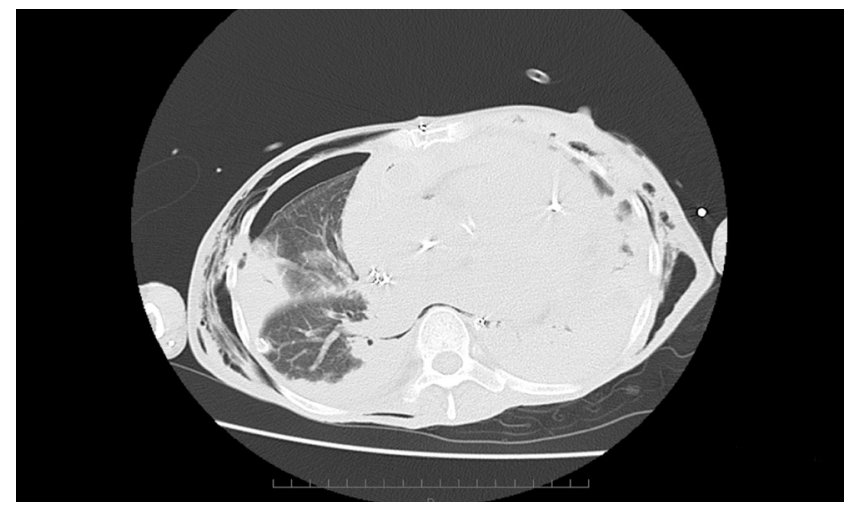

Figure 4. Computed tomogram immediately after the second intubation with extracorporeal membrane oxygenation showed pneumothorax and subcutaneous emphysema.

obstruction by a ball-valve clot, not tension pneumothorax.

A follow-up head CT revealed progression of whole hypoxic changes of the brain. Although VA-ECMO was established, severe hypoxia and hypoperfusion might have caused encephalopathy. Supportive care was continued, and the patient died 2 weeks later.

\section{DISCUSSION}

In the present case, cardiac arrest was not observed during the resuscitation period, but the patient showed signs of fatal brain hypoxia and finally died. Although HeartMate II continued to operate, the significant increase in intrathoracic pressure may have led to extreme heart failure and insufficient perfusion.

Tube obstruction should be considered an important factor of dislodgement of the tube, obstruction, pneumothorax, and equipment in the intubated patient with a sudden deterioration of ventilation [9]. Dislodgement of the endotracheal tube might occur during patient care such as body position changes, bathing, and rehabilitation. Intensivists and staffs much be very careful not to unintentionally dislocate a chest tube. The present intubation was performed by the senior experienced anesthesiologist, and human error was not suspected.

Immediately after intubation, the care team including the senior anesthesiologists confirmed safe intubation and ventilation, including detection of expired carbon dioxide. However, ventilation became more difficult within three minutes. Initially, the Jackson-Rees circuit completely was functional, with no equipment failure. The emergency thoracenteses slightly but significantly increased the arterial blood pressure and decreased the pulmonary artery and central venous pressures.
Relapsed pneumothorax was considered, and the results of a diagnostic thoracentesis were consistent with aggravating tension pneumothorax. Actually, a large clot formed in the trachea and, within a few hours, obstructed the tube tip as if it were a one-way valve. Immediately after intubation, inspiration was only possible with the high pressure of manual bag ventilation.

The patient's chest tube had been removed the day before the event. Her history of preceding pneumothorax likely influenced the decision-making process leading up to the complication. Moreover, pulmonary artery and central venous pressures suggested progression of tension pneumothorax. Mechanical obstruction of the endotracheal tube was considered unlikely. Placement of a Mini-Trach may have increased the difficulty of intubation. Thus, the intensivists used video laryngoscopy and prepared other devices including the intubating gum-elastic bougie.

Inoue et al. [2] reported a similar case of endotracheal tube obstruction due to a massive clot resulting from late bleeding with a mini-tracheostomy tube. The patient had received anticoagulants, and the massive blood clot may have formed over several days.

The values of prothrombin time-international normalization ratio and activated partial thrombin time in the presented case were 1.71 and 39.1 seconds, respectively. The results were appropriate for management of LVAD and were acceptable against the risk of hemorrhagic complications. However, we should have observed the changes in respiratory pattern after the intervention.

In summary, history of a preceding pneumothorax and increasing pulmonary artery and central venous pressures strongly suggest progression of tension pneumothorax. Although an urgent and rapid diagnosis of a ball-valve clot is not simple, intensivists should consider this rare and fatal complication.

\section{CONFLICT OF INTEREST}

No potential Conflict of Interest relevant to this article was reported.

\section{ORCID}

Hisaaki Munakata https://orcid.org/0000-0002-9111-5844

Michiko Higashi $\quad$ https://orcid.org/0000-0002-0410-2229

Takahiro Tamura https://orcid.org/0000-0001-6916-9988

Yushi Ueda Adachi https://orcid.org/0000-0001-8899-5708 


\section{AUTHOR CONTRIBUTIONS}

Conceptualization: TT, YUA. Data curation: HM, YUA. Project administration: MH, YUA. Visualization: HM, YUA. Writing original draft: HM, YUA. Writing - review \& editing: all authors.

\section{REFERENCES}

1. Nolan JD. Prehospital and resuscitative airway care: should the gold standard be reassessed? Curr Opin Crit Care 2001;7: 413-21.

2. Inoue $\mathrm{H}$, Ito J, Uchida $\mathrm{H}$, Morita $\mathrm{M}$, Masuda T, Yamaya $\mathrm{K}$, et al. Lower airway obstruction due to a massive clot resulting from late bleeding following mini-tracheostomy tube insertion and subsequent clot removal and re-intubation. JA Clin Rep 2017;3:16.

3. Liberman JS, Weigel W, Neal JM. Difficult ventilation after successful intubation in the emergency setting due to a ball valve clot. A Case Rep 2016;6:291-2.

4. Woittiez KJ, Woittiez AJ. Fatal endotracheal tube obstruction due to the ball valve effect. BMJ Case Rep 2015;2015:bcr20142 08189.

5. Neuburger PJ, Galloway AC, Zervos MD, Kanchuger MS. Case report: separation from cardiopulmonary bypass with a rigid bronchoscope airway after hemoptysis and bronchial impaction with clot. Anesth Analg 2012;114:89-92.

6. Kruczek ME, Hoff BH, Keszler BR, Smith RB. Blood clot resulting in ball-valve obstruction in the airway. Crit Care Med 1982;10:122-3.

7. Haramo A, Shiota N, Fukagawa A, Adachi Y, Nishiwaki K. A small piece of hematoma obstructed the tracheostomy cannula as a check valve. Clin J Anesth (Jpn) 2017;41:212-4.

8. Arney KL, Judson MA, Sahn SA. Airway obstruction arising from blood clot: three reports and a review of the literature. Chest 1999;115:293-300.

9. Johnson KM, Lehman RE. Acute management of the obstructed endotracheal tube. Respir Care 2012;57:1342-4. 